\title{
ELECTROELASTIC FIELD CONCENTRATION ON ELLIPTICAL PORES IN TEXTURED MEDIA
}

\author{
T. D. SHERMERGOR and V. B. YAKOVLEV \\ Institute of Electronic Technology, Moscow, Russia
}

(Received 24 October 1995)

\begin{abstract}
A method of calculating the tensor operator of the electroelastic field concentration in the vicinity of two kinds of inhomogeneities (pores and crystalline inclusions) is developed. It is assumed that the shape of inhomogeneities is an ellipsoid. The materials of the matrix and/or inclusions have piezoelectric properties. Effective piezoelectric properties of textured polycrystalline media are calculated by means of the general singularity approximation of the theory of random fields. Methods for the analysis of the operators of electroelastic field concentration on the surface of the inclusion are used. The influence of the ellipsoidal pore shape on the tensor operators of electroelastic field concentration is considered. Data are shown in figures.
\end{abstract}

KEY WORDS: Effective properties, electroelastic field concentration, piezoelectricity, texture, pore.

\section{INTRODUCTION}

A real inhomogeneous material (a multicomponent polycrystal) has a very complex structure. It is defined by the size, shape, orientation of crystallographic axes of single crystallites and the presence of various kinds of defects: pores, microcracks, defects of packing, dislocations and others. The physical properties of polycrystals are defined by the effective characteristics - elastic, dielectric, piezoelectric and other ones. Thus, a polycrystalline material can have some anisotropy of properties that is defined by two factors. The first one is the primary axes orientation of single crystallites described by the orientation distribution function (ODF). And the second factor is the ordering of crystallite orientations when their shape is not spherical. One of the important problems related to the analysis of macroscopic properties is the study of local polycrystalline field characteristics. This problem is linked to the structure of inhomogeneities. It is well known that the values of electrical and mechanical fields in the vicinity of inhomogeneities can essentially differ from their average magnitudes in the material as a whole. Such defects as pores and microcracks can result in violations of the material continuity and perhaps be the reason of destruction. Therefore evaluation of the local electrical and mechanical fields in the vicinity of crystallites or microcracks is of practical interest.

The standard model of the inhomogeneity is an ellipsoid. Being a surface of second order this form of inhomogeneity permits to execute an analytical evaluation of the mechanical and electrical field concentration tensors in the vicinity of the inhomogeneity surface (Shermergor and Yakovlev 1993, Hill 1983, Kolker and other 1992, Kunin and Sosnina 1973). We shall emphasize the presence of anisotropy in the material. Because of this the location of the extremum values of the stress concentration can differ from those in an isotropic material (Kolker and other 1992). 
One of the methods to get information about the structure of a polycrystalline material is to study its reaction to external loads of mechanical pulses or harmonic elastic loads. And in piezoelectrics there is an additional possibility to study the electrical field distribution on the surface of a material subjected to the effect of mechanical loads. The analysis of the electrical field distributions on the surface of piezoelectric polycrystalline materials that undergo some external mechanical stress can give valuable information about the shape of inhomogeneous elements, too. For this purpose it is necessary first to determine the effective characteristics of a textured polycrystalline material. Then considering the whole material without a pore as a homogeneous one we find distributions of electroelastic fields in the vicinity of the pore surface (Shermergor and Yakovlev 1993). We shall analyze piezoelectric polycrystals.

\section{METHOD}

Local field characteristics have usually maximum deviations from their average values in the vicinity of a border inhomogeneity. A tensor operator of the electroelastic field concentration is commonly used in this case for the analysis of the local fields. This operator is defined by the relation between the local and external electrical and/or mechanical fields

$$
T_{i j}(\vec{n})=K_{i j k l}(\vec{n}) T_{k l}^{o}
$$

Here we used the following notations: $T_{i j}(\vec{n})$ and $T_{k l}^{o}$ are the local and external generalized stress correspondingly, $\vec{n}$ is the vector of the normal to the crystallite or pore surface (Hill 1983).

We define the generalized stress using the constitutional equations of piezoelectric media

$$
\begin{aligned}
& \sigma_{\mathrm{ij}}=\mathrm{c}_{\mathrm{ijk} l} \varepsilon_{\mathrm{k} 1}-\mathrm{e}_{\mathrm{kij}} E_{\mathrm{k}} \\
& \mathrm{D}_{\mathrm{i}}=\mathrm{e}_{\mathrm{ik} l} \varepsilon_{\mathrm{k} 1}+\beta_{\mathrm{ik}} E_{\mathrm{k}}
\end{aligned}
$$

Here the values $\sigma_{\mathrm{ij}}, \varepsilon_{\mathrm{k} 1}$ denote the tensors of mechanical stress and strain, and $\vec{D}, \vec{E}$ are the vectors of induction and intensity of an electrical field, $c_{\mathrm{ijkl}}, \mathrm{e}_{\mathrm{kij}}, \beta_{\mathrm{ik}}$ are the tensors of elasticity, permittivity and piezomuduli. In this connection the tensors of generalized stress $\mathrm{T}$, strain $\mathrm{Z}$ and elasticity modules $\mathrm{C}$ are defined in matrix notation by the following expressions

$$
T=\left[\begin{array}{c}
\sigma_{i j} \\
D_{i}
\end{array}\right], \quad Z=\left[\begin{array}{c}
\varepsilon_{k 1} \\
-E_{k}
\end{array}\right], \quad C=\left[\begin{array}{cc}
c_{i j k l} & e_{k i j} \\
e_{i k 1} & \beta_{k}
\end{array}\right] .
$$

In this notation the expressions (2) and (3) can be written in the following form

$$
\mathrm{T}=\mathrm{CZ}
$$

The solution of the problem to determine the electroelastic field concentration operator is possible only for a single inhomogeneity in a surrounding homogeneous medium which is, in the general case, anisotropic. The solution of this problem for the set of 
inhomogeneities results in a well-known problem of multiple bodies. The hypothesis of effective media is used for the analysis of local field characteristics of inhomogeneous materials. Effective media is the idealization of real inhomogeneous media. Its properties are described by effective properties of textured polycrystals (Walther and other 1991, Shermergor and other 1993, Bunge 1993).

The knowledge of a quantitative measure of the texture (ODF) is required to calculate the effective characteristics of polycrystals (Bunge 1993). Then, for example, by the method of general singularity approximation of the theory of random fields with the available ODF, it is possible to calculate the effective elastic, dielectric and piezoelectric material characteristics of polycrystals. These characteristics will determine the properties of the effective media.

If crystallites differ only in the orientation of the crystallographic axes we have the following expression for calculating the effective piezoelectric properties (without subscript) (Shermergor and others 1993):

$$
\left(\mathrm{C}^{*}-\mathrm{B}\right)^{-1}=\left(1 / 8 \pi^{2}\right) \int_{0}^{\pi} \int_{0}^{2 \pi} \int_{0}^{2 \pi} \mathrm{f}\left(\varphi_{1}, \Phi, \varphi_{2}\right)\left(\mathrm{C}\left(\varphi_{1}, \Phi, \varphi_{2}\right)-\mathrm{B}\right)^{-1} \sin \Phi \mathrm{d} \Phi \mathrm{d} \varphi_{1} \mathrm{~d} \varphi_{2}
$$

We shall note, that the effective characteristics of polycrystalline materials in the general singularity approximation are determined relative to the main axes of all the tensors. Here $\mathrm{f}\left(\varphi_{1}, \Phi, \varphi_{2}\right)$ is the ODF, and $\varphi_{1}, \Phi, \varphi_{2}$ are the coordinates in the Euler's space, and the asterisk indicates effective values. The tensor B is determined by the following expression

$$
\mathrm{B}=\mathrm{C}^{\mathrm{c}}+\mathrm{g}^{-1}
$$

where $\mathrm{C}^{\mathrm{c}}$ is the tensor of the comparison body. The components of tensor $\mathrm{g}$ are

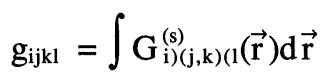

$\mathrm{G}_{\mathrm{i})(\mathrm{j}, \mathrm{k})(1(\overrightarrow{\mathrm{r}})}^{(\mathrm{s})}$ are the symetric symmetrization singular components of the second derivative of the Green's tensor of the balance equation of piezoelectric media.

We shall proceed to the direct analysis of local electroelastic fields. The main determining expression reads (Shermergor and Yakovlev 1993):

$$
K_{i j k 1}(\vec{n})=\left(C^{(2)}+C^{(2)} A(\vec{n}) C^{(3)}\right)\left(C^{(2)}+C^{(2)}\langle A(\vec{n})\rangle C^{(3)}\right)^{-1}
$$

where $\mathrm{C}^{(3)}=\mathrm{C}^{(2)}-\mathrm{C}^{(1)}$, and indices 1 and 2 correspond to the matrix and the pore. The tensor $A(\vec{n})$ is the tensor $G_{i)(j, k)(1(\vec{r})}^{(s)}$ in the Fourier space with $\vec{n}=\vec{k} /|\vec{k}|$, and $\langle A(\overrightarrow{\mathrm{n}})\rangle \equiv \mathrm{g}(8)$.

It is noted that the above operator depends on the physical and mechanical properties and anisotropy of the media and the inhomogeneity element, the kinds of external load and the shape of the inhomogeneity element. If the shape of the elements differs from the spherical one the orientation of the ellipsoidal pore will be an important factor determining the structure features.

\section{RESULTS AND DISCUSSION}

We shall consider an ellipsoidal vacuum pore placed in textured polycrystalline quartz. In this case the tensors of elasticity and piezomoduli of the pore are equal to zero 
and the tensor of permittivities is the tensor of vacuum. We assume that the volume concentration of the pores is small and thus they do not interact.

The effective piezoelectric characteristics of the polycrystalline textured media are calculated with help of the general singularity approximation of the theory of random fields. The ODF is determined by the Bunge's method (Bunge 1993) from the results of neutronographic experiments. The following effective values of the tensors of elasticity $(G P a)$, permittivities (relative values) and piezomoduli $\left(K l / \mathrm{m}^{2}\right)$ are obtained (points denote zero value):

$$
\begin{aligned}
& \mathrm{c}^{*}=\left[\begin{array}{rrrrrr}
96.6 & 8.3 & 8.3 & -0.2 & . & . \\
8.3 & 96.6 & 8.3 & 0.2 & . & . \\
8.3 & 8.3 & 96.6 & . & . & . \\
-0.2 & 0.2 & . & 44.2 & . & . \\
. & . & . & . & 44.2 & -0.2 \\
. & . & . & . & -0.2 & 44.2
\end{array}\right]
\end{aligned}
$$

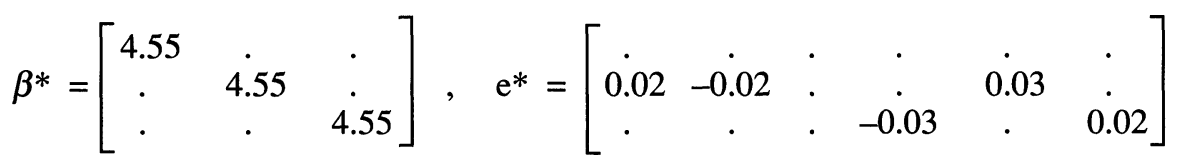

To define the kind of external mechanical loads, three kinds of a stress condition (axial, plane and volumetric) are of great interest for investigations. We shall be limited by considering an axial stress condition, for example, in the direction of the z-axis. Let the main axis of the ellipsoidal pore and external stress be also oriented in this direction. Moreover, the tensors of physical and mechanical characteristics in this direction are in the main axes.

We shall consider the change of the tensor operator of the electroelastic stress concentration caused by the variation of the main axis size of the ellipsoidal pore. The calculated data are indicated in Figure 1-2. The angles $\varphi$ and $\theta$ define the directions of the normal to the pore surface.

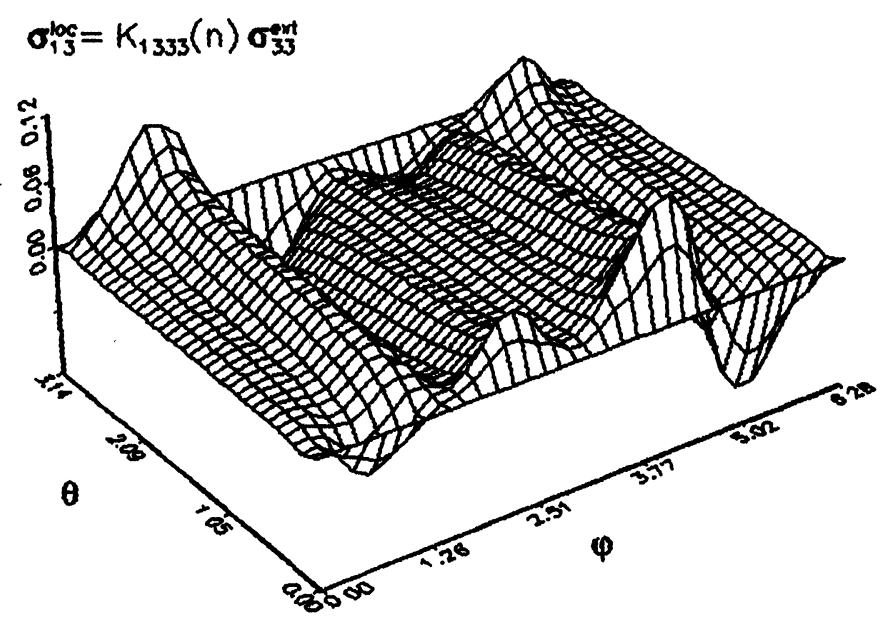

Figure 1a Distribution of the shear stress $\sigma_{13}$ on the surface of the pore, induced by the tensile stress $\sigma_{33}$ for a needle shaped pore $(\mathrm{c} / \mathrm{a}=100)$. 


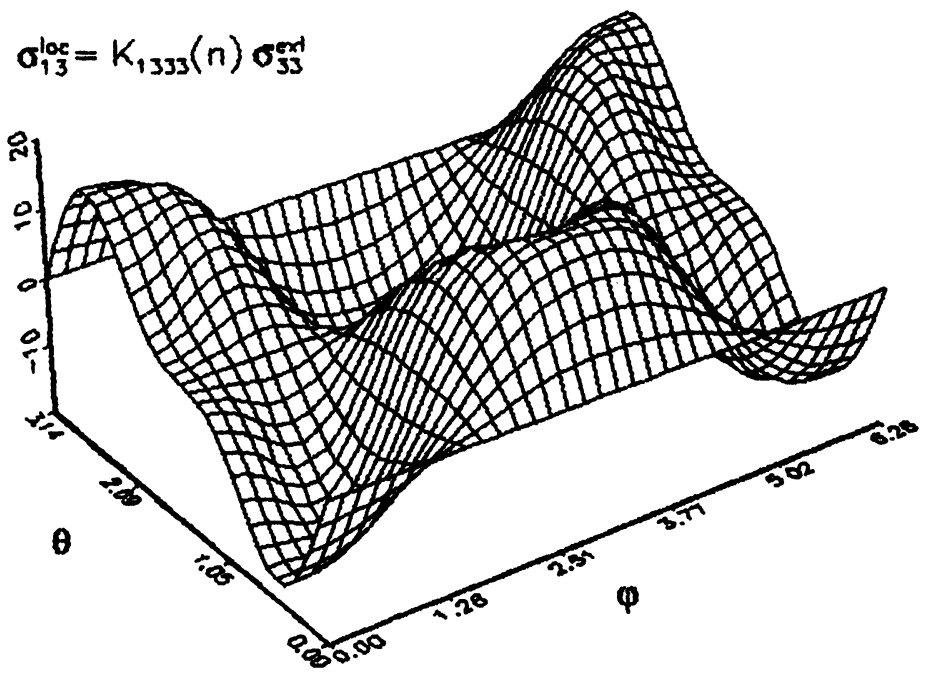

Figure $1 \mathrm{~b}$ Distribution of the shear stress $\sigma_{13}$ on the surface of the pore, induced by the tensile stress $\sigma_{13}$ for a sphere shaped pore.

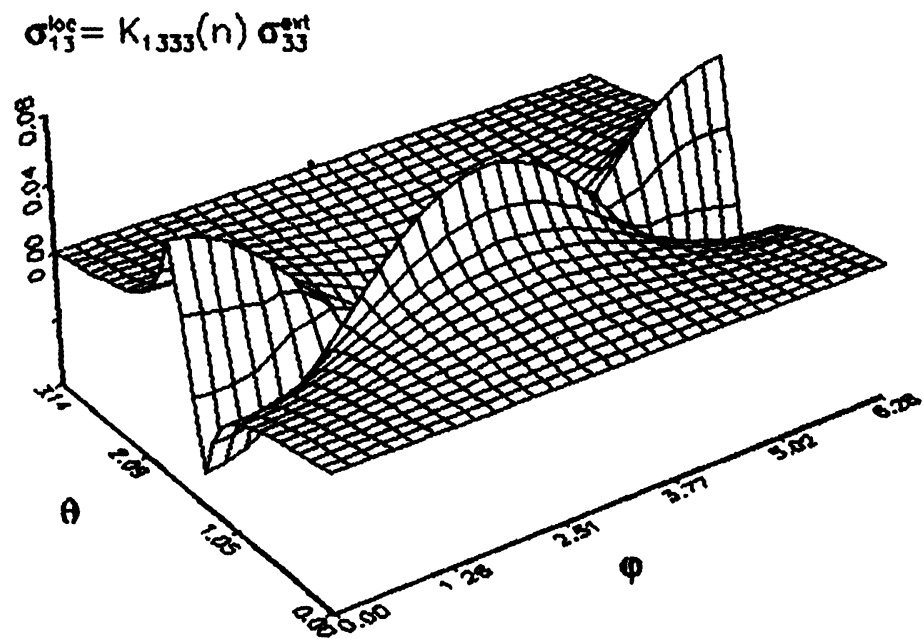

Figure 1c Distribution of the shear stress $\sigma_{13}$ on the surface of the pore, induced by the tensile stress $\sigma_{33}$ for a disk shaped pore $(\mathrm{c} / \mathrm{a}=\mathbf{0 . 0 1})$.

In Figure 1 the coefficient $K_{1333}(\vec{n})$ of the elastic field concentration operator is indicated. It relates the values of the shear stress $\sigma_{13}$ on the pore surface and the external average tensile stress $\sigma_{33}$. The graph in Figure 1a corresponds to a needle elliptic pore with a ratio of axes: $\mathbf{b} / \mathbf{a}=\mathbf{1}, \mathbf{c} / \mathbf{a}=100$; in Figure $1 \mathrm{~b}$ to a spherical pore $(\mathbf{b} / \mathbf{a}=\mathbf{c} / \mathbf{a}=\mathbf{1})$ and in Figure $1 \mathrm{c}$ to $\mathbf{a}$ disk elliptic pore $(\mathbf{b} / \mathbf{a}=\mathbf{1}, \mathbf{c} / \mathbf{a}=\mathbf{0 . 0 1})$. It can be seen that in all the three cases the shear stresses on both ends of the ellipsoid $(\vartheta=0, \vartheta=\pi)$ at any angle $\varphi$ are equal to zero. The maximum of the absolute stress 
value for a needle pore appears to be shifted away from the poles of the ellipsoid. For a disk pore on the greater part of the disk surface the shear stress is absent at all. The maximum absolute values of the local stress $\sigma_{13}$ occur in the area near to edges $(\vartheta=\pi / 2)$. Thus, the change of the angle $\varphi$ from 0 to $2 \pi$ is accompanied by the change of the sign of the shear stress. When the shape of the pore changes from needle to a disk the character of the shear stress distribution changes essentially. So, for a needle pore the maximum absolute value of the shear stress corresponds to $\varphi=(\pi / 2),(2 \pi-\pi / 2)$, and for a disk pore the maximum absolute value of the shear stress correspond to angles $\varphi=0, \pi$. A little unexpected was the complete absence of the shear stress at any values of the angle $\vartheta$ when $\varphi=0,2 \pi$. Such a distribution of the shear stress is probably related to the character of quartz anisotropy.

In Figure 2 the values of the electrical induction $\mathbf{D}_{2}$ resulting from the action of the external tensile stress $\sigma_{33}$ are indicated. It is seen that in all cases the induction has the same sign. The distribution of induction for the needle (Figure 2a) and spherical (Figure 2b) pore has a similar character. But for the needle pore the maximas are expressed more sharply. For a disk pore (Figure 2c) the same maxima are displayed in the vicinity of the angles $\vartheta=\pi / 2$. In other areas of the pore surface the electrical induction $\mathbf{D}_{2}$ is absent at all.

\section{CONCLUSIONS}

Analysis of the pictures enables us to make the following basic conclusions:

1. The shape of the pore has an essential influence on the distribution of the stresses in the pore surface.

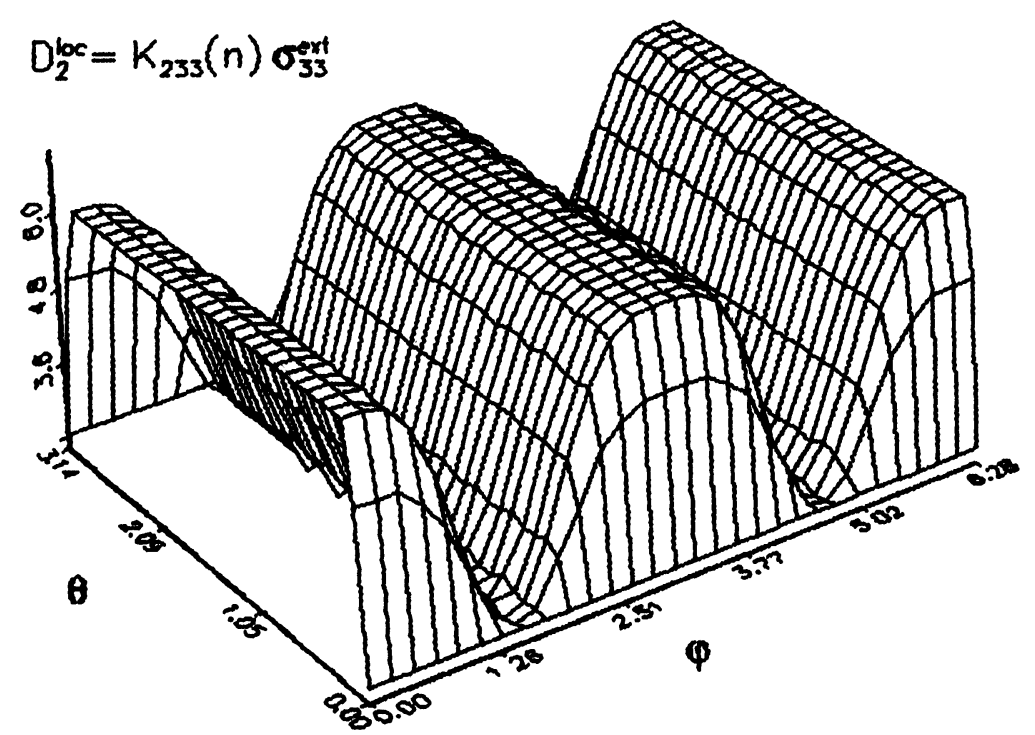

Figure 2a Distribution of the electrical induction $\mathbf{D}_{2}$ induced by the mechanical tensile stress $\sigma_{33}$ for a needle pore $(c / a=100)$. 


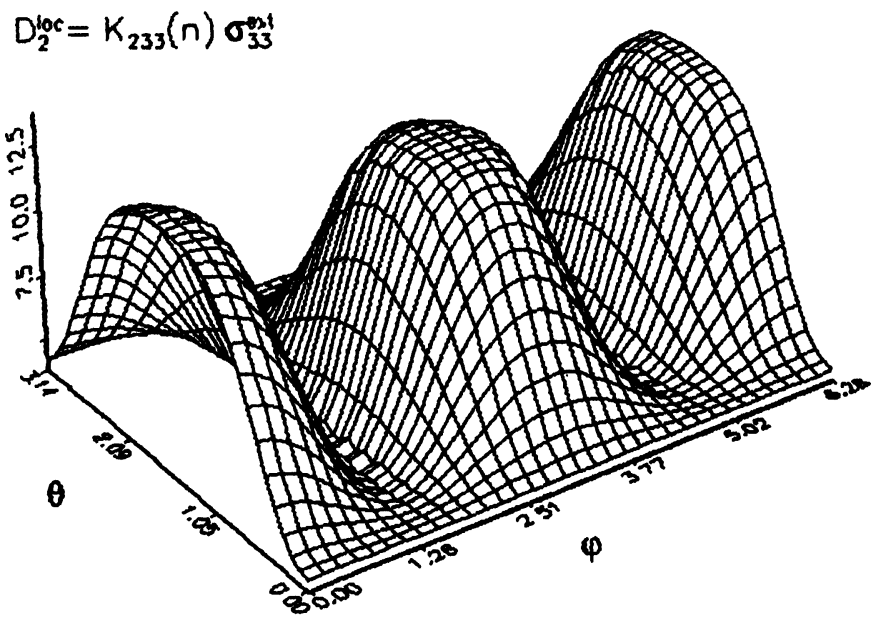

Figure 2b Distribution of the electrical induction $\mathbf{D}_{\mathbf{2}}$ induced by the mechanical tensile stress $\sigma_{33}$ for a spherical pore.

$$
D_{2}^{\text {koc }}=K_{233}(n) \sigma_{33}^{\text {ent }}
$$

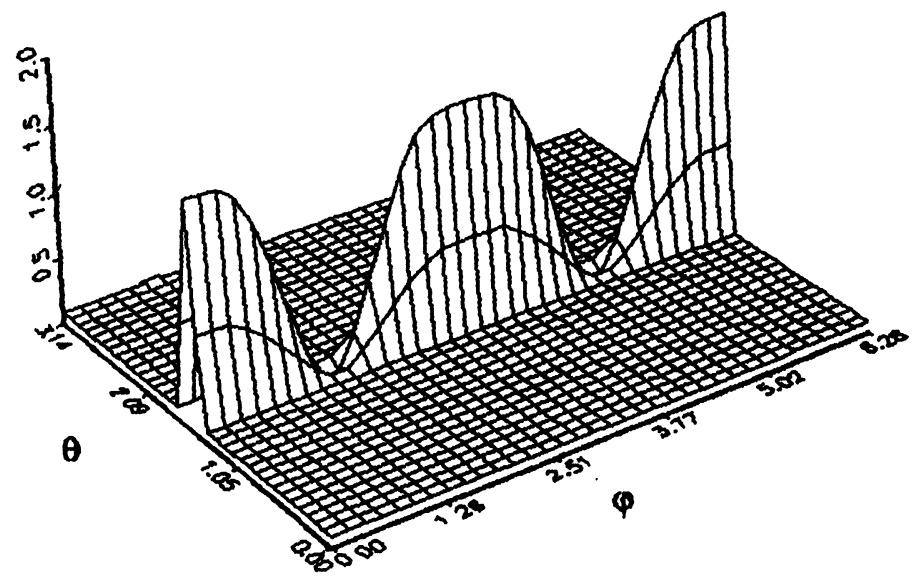

Figure 2c Distribution of the electrical induction $\mathbf{D}_{2}$ induced by the mechanical tensile stress $\sigma_{33}$ for disk pore $(\mathrm{c} / \mathrm{a}=\mathbf{0 . 0 1})$.

2. Changes of the pore shape when its volume is constant result in changes of the electroelastic field concentration values.

3. Extrema determine the critical areas that can for example initiate microdestruction.

\section{Acknowledgement}

The research described in this publication was made possible in part by grant No. JGG100 from the International Science Foundation and Russian Government. 


\section{References}

Bunge H. J. (1993). Texture Analysis in Material Science. Cuvillior, Gottingen 330.

Hill R. J. (1983). Interfacial Operators in the Mechanics of Composite Media.// Mech. Phys. Solids, 31, 347-357.

Kolker L. F., Mirenkova G. N. and Soanina E. G. (1992). Dependents of Stress on the Surface of Cracks from Media Anisotropy.// J. of Appl. Mech. and Tech. Phys., 196, 121-126. (in Russian).

Kunin I. A. and Soanina E. G. (1973). Stress Concentration on Elliptic Inhomogeneity in Anisotropic Media.// J. Appl. Math. Mech., 37, 306-315. (in Russian).

Shermergor T. D., Nikitin A. N., Walther K., Voitus, W. Ivankina T. I. and Yakovlev V. B. (1993). Determination of effective electroelastic constants of textured polycrystal rocks.// Izvestiya Akademii Nauk Russia, seriya Physics of the Earth (English edition). 2, 83-88.

Shermergor T. D. and Yakovlev V. B. (1993). Concentration of coupled electromechanical fields on the surface of a crystallite in textured polycrystal quartz.// Izvestiya Akademii Nauk Russia, seriya Physics of the Earth (English edition). 2, 89-94.

Walther K., Ivankina T. I., Nikitin A. N., Voitus, W. Shermergor T. D. and Yakovlev V. B. (1991). The determination of the effective physical characteristics of anisotropic geomaterials on a data of texture analysis.// Doklady Akademii Nauk USSR (English edition), 319, 310-314. 